\title{
Complexity of Strong Implementability
}

\author{
Clemens Thielen and Sven O. Krumke \\ Department of Mathematics, University of Kaiserslautern, Paul-Ehrlich-Str. 14, D-67663 Kaiserslautern, Germany \\ \{thielen, krumke\}@mathematik.uni-kl.de
}

\begin{abstract}
We consider the question of implementability of a social choice function in a classical setting where the preferences of finitely many selfish individuals with private information have to be aggregated towards a social choice. This is one of the central questions in mechanism design. If the concept of weak implementation is considered, the Revelation Principle states that one can restrict attention to truthful implementations and direct revelation mechanisms, which implies that implementability of a social choice function is "easy" to check. For the concept of strong implementation, however, the Revelation Principle becomes invalid, and the complexity of deciding whether a given social choice function is strongly implementable has been open so far. In this paper, we show by using methods from polyhedral theory that strong implementability of a social choice function can be decided in polynomial space and that each of the payments needed for strong implementation can always be chosen to be of polynomial encoding length. Moreover, we show that strong implementability of a social choice function involving only a single selfish individual can be decided in polynomial time via linear programming.
\end{abstract}

Keywords: computational complexity, algorithmic game theory, mechanism design, social choice

\section{Introduction}

Mechanism design is a classical area of noncooperative game theory [7] and microeconomics [2] which studies how privately known preferences of several people can be aggregated towards a social choice. Applications include the design of voting procedures, the writing of contracts among parties, and the construction of procedures for deciding upon public projects. Recently, the study of the Internet has fostered the interest in algorithmic aspects of mechanism design [6].

In the classical social choice setting considered in this paper, there are $n$ selfish agents, which must make a collective decision from some finite set $X$ of possible social choices. Each agent $i$ has a private value $\theta_{i} \in \Theta_{i}$ (called the agent's type), which influences the preferences of all agents over the alternatives in $X$. Formally, this is modeled by a valuation function $V_{i}: X \times \Theta \rightarrow \mathbb{Q}$ for each agent $i$, where $\Theta=$ $\Theta_{1} \times \cdots \times \Theta_{n}$. Every agent $i$ reports some information $s_{i}$ from a set $S_{i}$ of possible bids of $i$ to the mechanism designer who must then choose an alternative from $X$ based on these bids. The goal of the mechanism designer is to implement a given social choice function $f: \Theta \rightarrow X$, that is, to make sure that the alternative $f(\theta)$ is always chosen in equilibrium when the vector of true types is $\theta=\left(\theta_{1}, \ldots, \theta_{n}\right)$. To achieve this, the mechanism designer hands out a payment $P_{i}(\theta)$ to each agent $i$, which depends on the bids. Each agent then tries to maximize the sum of her valuation and payment by choosing an appropriate bid depending on her type. A mechanism $\Gamma=\left(S_{1}, \ldots, S_{n}, g, P\right)$ is defined by the sets $S_{1}, \ldots, S_{n}$ of possible bids of the agents, an outcome function $g: S_{1} \times \cdots \times S_{n} \rightarrow X$, and the payment scheme $P=\left(P_{1}, \ldots, P_{n}\right)$.

In the most common concept called weak implementation, the mechanism $\Gamma$ is said to implement the social choice function $f$ if some (Bayesian) equilibrium of the noncooperative game defined by the mechanism yields the outcome specified by $f$. An important result known as the Revelation Principle (cf. 
[2. p. 884]) states that a social choice function is weakly implementable if and only if it can be truthfully implemented by a direct revelation mechanism, which means that $f$ can be implemented by a mechanism with $S_{i}=\Theta_{i}$ for all $i$ and truthful reporting as an equilibrium that yields the outcome specified by $f$. As a result, the question whether there exists a mechanism that weakly implements a given social choice function $f$ can be easily answered in time polynomial in $|\Theta|$ by checking for negative cycles in complete directed graphs on the agents' type spaces with changes of valuations as edge weights (cf. [4]).

However, there is an obvious drawback in considering weak implementation: Although a mechanism $\Gamma$ may have some equilibrium that yields the outcome specified by $f$, there may be other equilibria that yield different outcomes. Thus, the concept of weak implementation heavily relies on the implicit assumption that the agents always play the equilibrium that the mechanism designer wants if there is more than one.

The standard way to avoid this problem is to consider the more robust concept of implementation called strong implementation. A mechanism $\Gamma$ is said to strongly implement the social choice function $f$ if every equilibrium of $\Gamma$ yields the outcome specified by $f$. For strong implementation, the Revelation Principle does not hold, so one cannot, in general, restrict attention to direct revelation mechanisms and truthful implementations when trying to decide whether a social choice function is strongly implementable. In tackling the question whether a given social choice function can be strongly implemented, it is not even a priori clear that one can restrict attention to finite sets $S_{i}$ or polynomially sized payments. Thus, to the best of our knowledge, the complexity has been open so far. The main result of this paper is that strong implementability of a social choice function can be decided in polynomial space. In particular, if a social choice function can be strongly implemented, our results show that each of the payments in a mechanism that strongly implements it can be chosen to be of polynomial encoding length. It seems unlikely that the problem is contained in NP, at least the characterizations of strong implementability developed so far require an exponential number of (polynomially sized) certificates. We conjecture that deciding strong implementability is in fact PSPACE-complete. However, we show that the problem can be solved in polynomial time in case of a single agent.

Problem Definition We are given $n$ agents identified with the set $N=\{1, \ldots, n\}$ and a finite set $X$ of possible social choices. For each agent $i$, there is a finite set $\Theta_{i}$ of possible types and we write $\Theta=\Theta_{1} \times \cdots \times \Theta_{n}$. The true type $\theta_{i}$ of agent $i$ is known only to the agent herself. However, there is a commonly known probability distribution $p: \Theta \rightarrow \mathbb{Q}$ on $\Theta$ satisfying $p(\theta) \geq 0$ for all $\theta \in \Theta$ and $\sum_{\theta \in \Theta} p(\theta)=1$. Thus, every agent knows the probabilities of all possible vectors $\theta \in \Theta$ of types of all agents. Without loss of generality, we assume the marginal probability $p_{i}\left(\theta_{i}\right):=\sum_{\theta_{-i} \in \Theta_{-i}} p\left(\theta_{-i}, \theta_{i}\right)$ to be strictly positive for every $\theta_{i} \in \Theta_{i}$. The beliefs of an agent $i$ of type $\theta_{i}$ are then given by the (conditioned) probability distribution $q_{i}\left(. \mid \theta_{i}\right)$ over $\Theta_{-i}$ defined by

$$
q_{i}\left(\theta_{-i} \mid \theta_{i}\right):=\frac{p\left(\theta_{-i}, \theta_{i}\right)}{p_{i}\left(\theta_{i}\right)} .
$$

All agents as well as the mechanism designer know these beliefs for every $i$ and every $\theta_{i}$ since they know the probability distribution $p$. However, as mentioned above, only agent $i$ knows the true value of $\theta_{i}$.

Each agent $i$ has a valuation function $V_{i}: X \times \Theta \rightarrow \mathbb{Q}$, where $V_{i}(x, \theta)$ specifies the value that agent $i$ assigns to alternative $x \in X$ when the types of the agents are $\theta \in \Theta$. A social choice function in this setting is a function $f: \Theta \rightarrow X$ that assigns an alternative $f(\theta) \in X$ to every vector $\theta$ of types.

Definition 1. A mechanism $\Gamma=\left(S_{1}, \ldots, S_{n}, g, P\right)$ consists of a set $S_{i}$ of possible bids for each agent $i$, an outcome function $g: S \rightarrow X$ and a payment scheme $P: S \rightarrow \mathbb{Q}^{n}$, where $S:=S_{1} \times \cdots \times S_{n}$. $\Gamma$ is called $a$ direct revelation mechanism if $S_{i}=\Theta_{i}$ for all $i \in N$. We denote the direct revelation mechanism $\left(\Theta_{1}, \ldots, \Theta_{n}, f, P\right)$ defined by a social choice function $f$ and a payment scheme $P$ by $\Gamma_{(f, P)}$. 
A strategy for agent $i$ in the mechanism $\Gamma$ is a function $\alpha_{i}: \Theta_{i} \rightarrow S_{i}$ that defines a bid $\alpha_{i}\left(\theta_{i}\right) \in S_{i}$ for every possible type $\theta_{i}$ of agent $i$. A strategy profile (in the mechanism $\Gamma$ ) is an $n$-tuple $\alpha=\left(\alpha_{1}, \ldots \alpha_{n}\right)$ containing a strategy $\alpha_{i}$ for each agent $i$.

Definition 2. Given a mechanism $\Gamma=\left(S_{1}, \ldots, S_{n}, g, P\right)$ and an $(n-1)$-tuple $\alpha_{-i}$ of strategies for all agents except $i$, the expected utility from a bid $s_{i} \in S_{i}$ for agent $i$ when her type is $\theta_{i}$ is defined as

$$
U_{i}^{\Gamma}\left(\alpha_{-i}, s_{i} \mid \theta_{i}\right):=\sum_{\theta_{-i} \in \Theta_{-i}} q_{i}\left(\theta_{-i} \mid \theta_{i}\right) \cdot\left(V_{i}\left(g\left(\alpha_{-i}\left(\theta_{-i}\right), s_{i}\right), \theta\right)+P_{i}\left(\alpha_{-i}\left(\theta_{-i}\right), s_{i}\right)\right)
$$

The strategy profile $\alpha=\left(\alpha_{1}, \ldots, \alpha_{n}\right)$ is a (Bayesian) equilibrium of $\Gamma$ if $\alpha_{i}\left(\theta_{i}\right)$ maximizes the expected utility of an agent $i$ of type $\theta_{i}$ for every $i \in N$ and every $\theta_{i} \in \Theta_{i}$, i.e., if $\alpha_{i}\left(\theta_{i}\right) \in \operatorname{argmax}_{s_{i} \in S_{i}} U_{i}^{\Gamma}\left(\alpha_{-i}, s_{i} \mid \theta_{i}\right)$ for all $i \in N, \theta_{i} \in \Theta_{i}$. A direct revelation mechanism $\Gamma_{(f, P)}$ is called incentive compatible if truthful reporting is an equilibrium.

Note that incentive compatibility of a mechanism is often defined as the property that truthful reporting is a dominant strategy equilibrium. However, since we consider Bayesian equilibria, the definition given above is the natural choice in our setting.

Definition 3. The mechanism $\Gamma=\left(S_{1}, \ldots, S_{n}, g, P\right)$ strongly implements the social choice function $f$ if

1. $\Gamma$ has at least one equilibrium,

2. Every equilibrium $\alpha$ of $\Gamma$ satisfies $g \circ \alpha=f$.

The social choice function $f$ is called strongly implementable if there exists a mechanism $\Gamma$ that strongly implements $f$.

Definition 4 (Strong Implementability Problem).

INSTANCE: The number $n$ of agents, the set $X$ of possible social choices, the sets $\Theta_{i}$ of possible types of the agents, the valuation functions $V_{i}: X \times \Theta \rightarrow \mathbb{Q}$, the probability distribution $p$ over $\Theta$, and the social choice function $f: \Theta \rightarrow X$.

QUESTION: Is $f$ strongly implementable?

To encode an instance of Strong Implementability, we need to do the following: For every valuation function $V_{i}: X \times \Theta \rightarrow \mathbb{Q}$, we need to store $|X| \cdot|\Theta|$ rational numbers. For the probability distribution $p$ : $\Theta \rightarrow \mathbb{Q}$, we need to store $|\Theta|$ nonnegative rational numbers. The social choice function $f: \Theta \rightarrow X$ has encoding length $|\Theta| \cdot \log (|X|)$. Thus, the encoding length of an instance of Strong Implementability is in $\Omega(|X| \cdot|\Theta| \cdot n)$.

Observe that, in general, the encoding length is exponential in the number $n$ of agents, since, even when each agent has just two possible types, we have $|\Theta|=2^{n}$. It remains an interesting question for future research to study the problem and its complexity if the valuation functions $V_{i}$, the probability distribution $p$, and the social choice function $f$ are not explicitly specified but can only be accessed via an oracle. In this case, one would be interested in the existence/non-existence of oracle-polynomial time/space algorithms. We conjecture that exponential lower bounds can be proved in this setting.

\section{Solvability in Polynomial Space}

In this section, we show that Strong Implementability is contained in the complexity class PSPACE. One of the key ingredients of our argumentation is a result due to Mookherjee and Reichelstein [3] called the Augmented Revelation Principle. The mechanism design setting considered in [3] is similar to ours. The proof of the Augmented Revelation Principle given in [3] is focused on the case where no payments are allowed in a mechanism. We now give a proof for our setting of mechanisms with payments. 
Definition 5. A mechanism $\Gamma=\left(S_{1}, \ldots, S_{n}, g, P\right)$ is called augmented revelation mechanism if $S_{i}=\Theta_{i} \cup T_{i}$ for all $i \in N$ and arbitrary sets $T_{i}$.

In the above definition, the elements of the set $T_{i}$ represent additional bids available to agent $i$, in addition to her possible types.

Theorem 1 (The Augmented Revelation Principle).

If a social choice function $f: \Theta \rightarrow X$ is strongly implementable, then $f$ can be strongly implemented by an augmented revelation mechanism in which truthful reporting is an equilibrium.

Proof. Let $\Gamma=\left(S_{1}, \ldots, S_{n}, g, P\right)$ strongly implement $f$. We construct an augmented revelation mechanism $\bar{\Gamma}=\left(\bar{S}_{1}, \ldots, \bar{S}_{n}, \bar{g}, \bar{P}\right)$ that strongly implements $f$ as in the original proof of the Augmented Revelation Principle in [3]. However, we additionally have to define the new payment scheme $\bar{P}$ in terms of the given payment scheme $P$. Given an arbitrary equilibrium $\alpha=\left(\alpha_{1}, \ldots, \alpha_{n}\right)$ of $\Gamma$, we define $\bar{S}_{i}:=\Theta_{i} \cup T_{i}$, where

$$
T_{i}:=\left\{s_{i} \in S_{i} \mid s_{i} \notin \operatorname{image}\left(\alpha_{i}\right)\right\}
$$

and image $\left(\alpha_{i}\right)=\left\{\alpha_{i}\left(\theta_{i}\right) \mid \theta_{i} \in \Theta_{i}\right\}$ denotes the image of the function $\alpha_{i}: \Theta_{i} \rightarrow S_{i}$. We consider the functions $\phi_{i}: \bar{S}_{i} \rightarrow S_{i}$ given by

$$
\phi_{i}\left(\bar{s}_{i}\right):=\left\{\begin{array}{cc}
\alpha_{i}\left(\theta_{i}\right) & \text { if } \bar{s}_{i}=\theta_{i} \text { for } \theta_{i} \in \Theta_{i} \\
\bar{s}_{i} & \text { if } \bar{s}_{i} \in T_{i}
\end{array}\right.
$$

and define the outcome function $\bar{g}: \bar{S} \rightarrow X$ as $\bar{g}:=g \circ \phi$, where $\phi=\left(\phi_{1}, \ldots, \phi_{n}\right)$. The payment scheme $\bar{P}$ : $\bar{S} \rightarrow \mathbb{Q}$ is defined analogously as $\bar{P}:=P \circ \phi$.

To show that $\bar{\Gamma}$ strongly implements $f$, suppose that $\bar{\alpha}=\left(\bar{\alpha}_{1}, \ldots, \bar{\alpha}_{n}\right)$ is an equilibrium of $\bar{\Gamma}$ and consider the the strategy profile $\alpha^{*}=\left(\alpha_{1}^{*}, \ldots, \alpha_{n}^{*}\right)$ in $\Gamma$ given by $\alpha_{i}^{*}:=\phi_{i} \circ \bar{\alpha}_{i}$. By definition of $\alpha^{*}, \bar{g}$, and $\bar{P}$ we then have $g \circ \alpha^{*}=g \circ \phi \circ \bar{\alpha}=\bar{g} \circ \bar{\alpha}$ and $P \circ \alpha^{*}=P \circ \phi \circ \bar{\alpha}=\bar{P} \circ \bar{\alpha}$. We claim that $\alpha^{*}$ is an equilibrium of $\Gamma$. Since every $\phi_{i}: \bar{S}_{i} \rightarrow S_{i}$ is surjective, we can choose, for every fixed $s_{i} \in S_{i}$, an element $\bar{s}_{i} \in \bar{S}_{i}$ with $\phi_{i}\left(\bar{s}_{i}\right)=s_{i}$. Then, for every $i \in N$ and every possible $\theta_{i} \in \Theta_{i}$, we have

$$
\begin{aligned}
U_{i}^{\Gamma}\left(\alpha_{-i}^{*}, \alpha_{i}^{*}\left(\theta_{i}\right) \mid \theta_{i}\right) & =\sum_{\theta_{-i} \in \Theta_{-i}} q_{i}\left(\theta_{-i} \mid \theta_{i}\right)\left(V_{i}\left(g\left(\alpha^{*}(\theta)\right), \theta\right)+P_{i}\left(\alpha^{*}(\theta)\right)\right) \\
& =\sum_{\theta_{-i} \in \Theta_{-i}} q_{i}\left(\theta_{-i} \mid \theta_{i}\right)\left(V_{i}(\bar{g}(\bar{\alpha}(\theta)), \theta)+\bar{P}_{i}(\bar{\alpha}(\theta))\right) \\
& \geq \sum_{\theta_{-i} \in \Theta_{-i}} q_{i}\left(\theta_{-i} \mid \theta_{i}\right)\left(V_{i}\left(\bar{g}\left(\bar{\alpha}_{-i}\left(\theta_{-i}\right), \bar{s}_{i}\right), \theta\right)+\bar{P}_{i}\left(\bar{\alpha}_{-i}\left(\theta_{-i}\right), \bar{s}_{i}\right)\right) \\
& =\sum_{\theta_{-i} \in \Theta_{-i}} q_{i}\left(\theta_{-i} \mid \theta_{i}\right)\left(V_{i}\left(g\left(\alpha_{-i}^{*}\left(\theta_{-i}\right), \phi_{i}\left(\bar{s}_{i}\right)\right), \theta\right)+P_{i}\left(\alpha_{-i}^{*}\left(\theta_{-i}\right), \phi_{i}\left(\bar{s}_{i}\right)\right)\right) \\
& =\sum_{\theta_{-i} \in \Theta_{-i}} q_{i}\left(\theta_{-i} \mid \theta_{i}\right)\left(V_{i}\left(g\left(\alpha_{-i}^{*}\left(\theta_{-i}\right), s_{i}\right), \theta\right)+P_{i}\left(\alpha_{-i}^{*}\left(\theta_{-i}\right), s_{i}\right)\right) \\
& =U_{i}^{\Gamma}\left(\alpha_{-i}^{*}, s_{i} \mid \theta_{i}\right)
\end{aligned}
$$

where the inequality follows since $\bar{\alpha}$ is an equilibrium of $\bar{\Gamma}$. Since $s_{i} \in S_{i}$ was arbitrary, this shows that $\alpha^{*}$ is an equilibrium of $\Gamma$ as claimed. So since $\Gamma$ strongly implements $f$, it follows that $f=g \circ \alpha^{*}=\bar{g} \circ \bar{\alpha}$, i.e., the equilibrium $\bar{\alpha}$ yields the outcome specified by $f$. Hence, it just remains to show that truthful bidding is an equilibrium of $\bar{\Gamma}$. But this follows easily since, for every $\theta \in \Theta$, we have $\bar{g}(\theta)=(g \circ \phi)(\theta)=$ $g(\alpha(\theta))$ and $\bar{P}(\theta)=(P \circ \phi)(\theta)=P(\alpha(\theta))$ and $\alpha$ is an equilibrium of $\Gamma$. 
We can now proceed analogously to [3] to show how the Augmented Revelation Principle can be used to obtain a necessary condition for strong implementability of a social choice function. Mookherjee and Reichelstein already proved that this condition is sufficient for mechanisms with payments, so we will in fact obtain a necessary and sufficient condition in our setting. Note, however, that the sufficiency result does not hold for mechanisms without payments. To formulate our necessary and sufficient condition for strong implementability, we need the following definitions:

Definition 6. An equilibrium $\alpha=\left(\alpha_{1}, \ldots, \alpha_{n}\right)$ in a direct revelation mechanism $\Gamma_{(f, P)}$ can be selectively eliminated if there exist an agent $i \in N$ and functions $h: \Theta_{-i} \rightarrow X$ and $\bar{P}_{i}: \Theta_{-i} \rightarrow \mathbb{Q}$ such that:

$$
\begin{aligned}
& \text { 1. for some } \bar{\theta}_{i} \in \Theta_{i}: \sum_{\theta_{-i} \in \Theta_{-i}} q_{i}\left(\theta_{-i} \mid \bar{\theta}_{i}\right) \cdot\left(V_{i}\left(h\left(\alpha_{-i}\left(\theta_{-i}\right)\right), \theta_{-i}, \bar{\theta}_{i}\right)+\bar{P}_{i}\left(\alpha_{-i}\left(\theta_{-i}\right)\right)\right) \\
& \qquad>\sum_{\theta_{-i} \in \Theta_{-i}} q_{i}\left(\theta_{-i} \mid \bar{\theta}_{i}\right) \cdot\left(V_{i}\left(f\left(\alpha_{-i}\left(\theta_{-i}\right), \alpha_{i}\left(\bar{\theta}_{i}\right)\right), \theta_{-i}, \bar{\theta}_{i}\right)+P_{i}\left(\alpha_{-i}\left(\theta_{-i}\right), \alpha_{i}\left(\bar{\theta}_{i}\right)\right)\right)
\end{aligned}
$$

In this definition, agent $i$ is given a new bid, which we will call a flag. When agent $i$ bids the flag and the other agents bid a vector $\theta_{-i} \in \Theta_{-i}$, the mechanism chooses the outcome $h\left(\theta_{-i}\right)$ and hands out the payment $\bar{P}_{i}\left(\theta_{-i}\right)$ to agent $i$. The first condition says that, for some type $\bar{\theta}_{i} \in \Theta_{i}$, agent $i$ can increase her expected utility by deviating from $\alpha_{i}$ to the flag. Thus, $\alpha$ is not an equilibrium anymore. However, the second condition ensures that agent $i$ can not increase her expected utility by deviating from truthful reporting to the flag in the case that all other agents bid their true types. Hence, truthful reporting is preserved as an equilibrium.

Definition 7. An equilibrium $\alpha$ of the direct revelation mechanism $\Gamma_{(f, P)}$ is called bad if $f \circ \alpha \neq f$. The direct revelation mechanism $\Gamma_{(f, P)}$ satisfies the selective elimination condition if every bad equilibrium $\alpha$ can be selectively eliminated.

Theorem 2. Suppose that the social choice function $f: \Theta \rightarrow X$ is strongly implementable. Then there exists an incentive compatible direct revelation mechanism $\Gamma_{(f, P)}$ that satisfies the selective elimination condition.

Proof. By Theorem 11 there exists an augmented revelation mechanism $\Gamma=\left(S_{1}, \ldots, S_{n}, g, P\right)$ that strongly implements $f$ and in which truthful reporting is an equilibrium. In particular, this implies that $g_{\mid \Theta}=f$. We now show that the direct revelation mechanism $\Gamma_{\left(f, P_{\Theta}\right)}$ is incentive compatible and satisfies the selective elimination condition. Incentive compatibility follows directly from the fact that truthful reporting is an equilibrium in $\Gamma$. Moreover, any bad equilibrium $\alpha$ of $\Gamma_{\left(f, P_{\Theta}\right)}$ can not be an equilibrium of $\Gamma$ since this would contradict the fact that $\Gamma$ strongly implements $f$. Hence, in $\Gamma$ there must be a non-type message available to some agent $i$ to which $i$ prefers to deviate when $\alpha$ is being played, without being tempted to do the same when all agents report truthfully. Thus, any bad equilibrium $\alpha$ can be selectively eliminated.

Mookherjee and Reichelstein [3] already proved that the condition from Theorem 2] is also sufficient for strong implementability of a social choice function $f$ in settings where payments are allowed. The idea of the proof is to start with an incentive compatible direct revelation mechanism and eliminate the finitely many bad equilibria one after another in order to obtain an augmented revelation mechanism that strongly implements $f$. However, one has to make sure that the augmentations do not induce new (bad) equilibria. This is done by giving additional bids (called counterflags) to some agent $j \neq i$ to make sure that the newly introduced flag of agent $i$ can never be used in an equilibrium. To achieve this, the payment scheme has to be modified appropriately. Together with Theorem 2 , the result of Mookherjee and Reichelstein [3] proves: 
Theorem 3. The social choice function $f: \Theta \rightarrow X$ is strongly implementable if and only if there exists an incentive compatible direct revelation mechanism $\Gamma_{(f, P)}$ that satisfies the selective elimination condition.

Theorem 3 is one of the keys to proving that the Strong Implementability Problem is in PSPACE (in fact, our proof shows that the problem is in NPSPACE, which equals PSPACE by Savitch's Theorem). The idea of our proof is to use the direct revelation mechanism $\Gamma_{(f, P)}$ and, for every bad equilibrium $\alpha$ of $\Gamma_{(f, P)}$, the index $i$, the pair of functions $\left(h, \bar{P}_{i}\right)$, and the type $\bar{\theta}_{i}$ needed to selectively eliminate $\alpha$, as a certificate for showing that $f$ is strongly implementable. The algorithm first guesses the polynomially many values $P_{i}(\theta)$ and then enumerates all possible strategy profiles $\alpha$ to check which are good or bad equilibria. If a bad equilibrium $\alpha$ is found, the algorithm guesses the data $\left(i, h, \bar{P}_{i}, \bar{\theta}_{i}\right)$ needed to selectively eliminate $\alpha$. However, in order to be able to run this algorithm in polynomial space, we have to prove that the certificates used in each step can be chosen to have only polynomial encoding length. In particular, we need to show that every value $P_{i}(\theta)$ of the payment functions and every value $\bar{P}_{i}\left(\theta_{-i}\right)$ can be chosen to have polynomial encoding length.

Theorem 4. The social choice function $f: \Theta \rightarrow X$ is strongly implementable if and only if there exists an incentive compatible direct revelation mechanism $\Gamma_{(f, P)}$ of polynomial encoding length that satisfies the selective elimination condition. In this case, for every (fixed) bad equilibrium $\alpha$, the data $\left(i, h, \bar{P}_{i}, \bar{\theta}_{i}\right)$ needed to selectively eliminate $\alpha$ can be chosen to have polynomial encoding length.

Note that Theorem 3 implies that, in order to prove the if and only if statement in the claim, we just have to prove that strong implementability of a social choice function $f: \Theta \rightarrow X$ implies the existence of a direct revelation mechanism with the given properties.

So assume that the social choice function $f$ is strongly implementable. Then, by Theorem 3 , there exists an incentive compatible direct revelation mechanism $\Gamma_{(f, P)}$ (of possibly more than polynomial encoding length) that satisfies the selective elimination condition. For each bad equilibrium $\alpha$ of $\Gamma_{(f, P)}$, we denote an index $i$, functions $h: \Theta_{-i} \rightarrow X$ and $\bar{P}_{i}: \Theta_{-i} \rightarrow \mathbb{Q}$, and a type $\bar{\theta}_{i} \in \Theta_{i}$ that can be used to selectively eliminate $\alpha$ by $i_{\alpha}, h^{\alpha}, \bar{P}_{i_{\alpha}}^{\alpha}$, and $\bar{\theta}_{i_{\alpha}}^{\alpha}$, respectively. Similarly, for every strategy profile $\alpha$ that is not an equilibrium, we denote an index $i$ and a pair $\left(\theta_{i}, \bar{\theta}_{i}\right)$ of types of agent $i$ such that $U_{i}^{\Gamma}\left(\alpha_{-i}, \bar{\theta}_{i} \mid \theta_{i}\right)>$ $U_{i}^{\Gamma}\left(\alpha_{-i}, \alpha_{i}\left(\theta_{i}\right) \mid \theta_{i}\right)$ by $i_{\alpha}$ and $\left(\theta_{i_{\alpha}}^{\alpha}, \bar{\theta}_{i_{\alpha}}^{\alpha}\right)$, respectively.

Note that the only part of the mechanism $\Gamma_{(f, P)}$ that could have more than polynomial encoding length is the payment scheme $P: \Theta \rightarrow \mathbb{Q}^{n}$, and, for every bad equilibrium $\alpha$, the only part of the data $\left(i_{\alpha}, h^{\alpha}, \bar{P}_{i_{\alpha}}^{\alpha}, \bar{\theta}_{i_{\alpha}}^{\alpha}\right)$ that could have more than polynomial encoding length is the function $\bar{P}_{i_{\alpha}}^{\alpha}$ : $\Theta_{-i_{\alpha}} \rightarrow \mathbb{Q}$. Hence, we only have to show that every value $P_{i}(\theta)$ of the payment functions and every value $\bar{P}_{i_{\alpha}}^{\alpha}\left(\theta_{-i_{\alpha}}\right)$ can be chosen to have polynomial encoding length.

To do so, we assume that we are given $i_{\alpha}, h^{\alpha}$, and $\bar{\theta}_{i_{\alpha}}^{\alpha}$ for every bad equilibrium $\alpha$ of $\Gamma_{(f, P)}$, and $i_{\alpha},\left(\theta_{i_{\alpha}}^{\alpha}, \bar{\theta}_{i_{\alpha}}^{\alpha}\right)$ for every strategy profile $\alpha$ that is not an equilibrium and consider the system of linear inequalities in the variables $P_{i}(\theta), \bar{P}_{i_{\alpha}}^{\alpha}\left(\theta_{-i_{\alpha}}\right)$, for $\theta \in \Theta, \theta_{-i_{\alpha}} \in \Theta_{-i_{\alpha}}$, displayed on page 7 .

Here, the inequalities (1) and (2) encode exactly which strategy profiles are equilibria of $\Gamma_{(f, P)}$ and (3), (4) correspond to conditions 1. and 2. in the definition of selective elimination of an equilibrium, respectively. Note that the number of inequalities and variables of the system is exponential in the size of the input of Strong Implementability. However, we know that the system has a solution given by the values $P_{i}(\theta), \bar{P}_{i_{\alpha}}^{\alpha}\left(\theta_{-i_{\alpha}}\right)$ given by the mechanism $\Gamma_{(f, P)}$ and the functions $\bar{P}_{i_{\alpha}}^{\alpha}$ specified by the selective elimination condition for the bad equilibria of $\Gamma_{(f, P)}$. Theorem 4 now follows immediately if we can prove the following result:

Proposition 1. The system of inequalities has a solution in which each component has polynomial encoding length. 
For all strategy profiles $\alpha$ that are not equilibria:

$$
\begin{gathered}
\sum_{\theta_{-i \alpha} \in \Theta_{-i \alpha}} q_{i_{\alpha}}\left(\theta_{-i_{\alpha}} \mid \theta_{i_{\alpha}}^{\alpha}\right) \cdot\left(V_{i_{\alpha}}\left(f\left(\alpha_{-i_{\alpha}}\left(\theta_{-i_{\alpha}}\right), \bar{\theta}_{i_{\alpha}}^{\alpha}\right), \theta_{-i_{\alpha}}, \theta_{i_{\alpha}}^{\alpha}\right)+P_{i_{\alpha}}\left(\alpha_{-i_{\alpha}}\left(\theta_{-i_{\alpha}}\right), \bar{\theta}_{i_{\alpha}}^{\alpha}\right)\right) \\
-\sum_{\theta_{-i \alpha} \in \Theta_{-i \alpha}} q_{i_{\alpha}}\left(\theta_{-i_{\alpha}} \mid \theta_{i_{\alpha}}^{\alpha}\right) \cdot\left(V_{i_{\alpha}}\left(f\left(\alpha_{-i_{\alpha}}\left(\theta_{-i_{\alpha}}\right), \alpha_{i_{\alpha}}\left(\theta_{i_{\alpha}}^{\alpha}\right)\right), \theta_{-i_{\alpha}}, \theta_{i_{\alpha}}^{\alpha}\right)+P_{i_{\alpha}}\left(\alpha_{-i_{\alpha}}\left(\theta_{-i_{\alpha}}\right), \alpha_{i_{\alpha}}\left(\theta_{i_{\alpha}}^{\alpha}\right)\right)\right)>0
\end{gathered}
$$

For all equilibria $\alpha$ and all $i \in N, \theta_{i}, \tilde{\theta}_{i} \in \Theta_{i}$ :

$$
\begin{aligned}
\sum_{\theta_{-i} \in \Theta_{-i}} & q_{i}\left(\theta_{-i} \mid \theta_{i}\right) \cdot\left(V_{i}\left(f\left(\alpha_{-i}\left(\theta_{-i}\right), \alpha_{i}\left(\theta_{i}\right)\right), \theta_{-i}, \theta_{i}\right)+P_{i}\left(\alpha_{-i}\left(\theta_{-i}\right), \alpha_{i}\left(\theta_{i}\right)\right)\right) \\
& -\sum_{\theta_{-i} \in \Theta_{-i}} q_{i}\left(\theta_{-i} \mid \theta_{i}\right) \cdot\left(V_{i}\left(f\left(\alpha_{-i}\left(\theta_{-i}\right), \tilde{\theta}_{i}\right), \theta_{-i}, \theta_{i}\right)+P_{i}\left(\alpha_{-i}\left(\theta_{-i}\right), \tilde{\theta}_{i}\right)\right) \geq 0
\end{aligned}
$$

For all bad equilibria $\alpha$ :

$$
\begin{aligned}
\sum_{\theta_{-i \alpha} \in \Theta_{-i \alpha}} q_{i_{\alpha}}\left(\theta_{-i_{\alpha}} \mid \bar{\theta}_{i_{\alpha}}^{\alpha}\right) \cdot\left(V_{i_{\alpha}}\left(h^{\alpha}\left(\alpha_{-i_{\alpha}}\left(\theta_{-i_{\alpha}}\right)\right), \theta_{-i_{\alpha}}, \bar{\theta}_{i_{\alpha}}^{\alpha}\right)+\bar{P}_{i_{\alpha}}^{\alpha}\left(\alpha_{-i_{\alpha}}\left(\theta_{-i_{\alpha}}\right)\right)\right) & \\
-\sum_{\theta_{-i \alpha} \in \Theta_{-i_{\alpha}}} q_{i_{\alpha}}\left(\theta_{-i_{\alpha}} \mid \bar{\theta}_{i_{\alpha}}^{\alpha}\right) \cdot\left(V_{i_{\alpha}}\left(f\left(\alpha_{-i_{\alpha}}\left(\theta_{-i_{\alpha}}\right), \alpha_{i_{\alpha}}\left(\bar{\theta}_{i_{\alpha}}^{\alpha}\right)\right), \theta_{-i_{\alpha}}, \bar{\theta}_{i_{\alpha}}\right)+P_{i_{\alpha}}\left(\alpha_{-i_{\alpha}}\left(\theta_{-i_{\alpha}}\right), \alpha_{i_{\alpha}}\left(\bar{\theta}_{i_{\alpha}}^{\alpha}\right)\right)\right) & >0
\end{aligned}
$$

For all bad equilibria $\alpha$ and all $\theta_{i_{\alpha}} \in \Theta_{i_{\alpha}}$ :

$$
\sum_{\theta_{-i \alpha} \in \Theta_{-i_{\alpha}}} q_{i_{\alpha}}\left(\theta_{-i_{\alpha}} \mid \theta_{i_{\alpha}}\right) \cdot\left(V_{i_{\alpha}}\left(f\left(\theta_{-i_{\alpha}}, \theta_{i_{\alpha}}\right), \theta_{-i_{\alpha}}, \theta_{i_{\alpha}}\right)+P_{i_{\alpha}}\left(\theta_{-i_{\alpha}}, \theta_{i_{\alpha}}\right)-V_{i_{\alpha}}\left(h^{\alpha}\left(\theta_{-i_{\alpha}}\right), \theta_{-i_{\alpha}}, \theta_{i_{\alpha}}\right)-\bar{P}_{i_{\alpha}}^{\alpha}\left(\theta_{-i_{\alpha}}\right)\right) \geq 0
$$


We let $\mathscr{P}$ denote the polyhedron defined by this system of inequalities when all strict inequalities are replaced by non-strict inequalities. In other words, $\mathscr{P}$ is the topological closure of the set of solutions of the system. We denote the number of bad equilibria by $p \in \mathbb{N}$, the number of strategy profiles that are not equilibria by $q \in \mathbb{N}$, and the total number of equilibria by $m \in \mathbb{N}$. Then, by multiplying all inequalities by -1 and rearranging, we can write $\mathscr{P}$ as

$$
\mathscr{P}=\left\{(x, y) \in \mathbb{R}^{n \cdot|\Theta|} \times \mathbb{R}^{l}: A x+B y \leq b\right\}
$$

where the vector $x \in \mathbb{R}^{n \cdot|\Theta|}$ represents the $n \cdot|\Theta|$ variables $P_{i}(\theta)$ and the vector $y \in \mathbb{R}^{l}$ represents the $l:=\sum_{\alpha \text { bad equ. }}\left|\Theta_{-i_{\alpha}}\right|$ variables $\bar{P}_{i_{\alpha}}^{\alpha}\left(\theta_{-i_{\alpha}}\right)$.

$A \in \operatorname{Mat}(k \times n \cdot|\Theta|, \mathbb{Q})$ and $B \in \operatorname{Mat}(k \times l, \mathbb{Q})$ are the matrices given by the coefficients of the variables $P_{i}(\theta)$ and $\bar{P}_{i_{\alpha}}^{\alpha}\left(\theta_{-i_{\alpha}}\right)$ in the system, respectively, where $k:=q+m \cdot \sum_{i=1}^{n}\left|\Theta_{i}\right|^{2}+p+\sum_{\alpha \text { bad equ. }}\left|\Theta_{i_{\alpha}}\right|$ is the total number of inequalities in the system. The vector $b \in \mathbb{Q}^{k}$ is given by the constant terms in the system, which are all (sums of) products of valuations and probabilities. In particular, each entry of the matrices $A, B$, and of the vector $b$ is of polynomial encoding length.

On our way to proving Proposition 1 and Theorem 4 we use some definitions and results from polyhedral theory. In particular, we use the fact that the vertex and facet complexity of a polyhedron are polynomially related to each other (see, e.g., [1]). The most common use in our context will be to conclude that if each inequality in a linear system (with potentially exponentially many inequalities) has polynomial encoding length, then there exists a solution of polynomial encoding length. We also use the property that the encoding length of the sum of $2^{\text {poly }(n)}$ many values $x_{i} \in \mathbb{Q}$ is bounded by a polynomial in $\operatorname{poly}(n)$ and the encoding sizes of the $x_{i}$.

Definition 8. The projection of a polyhedron $P \subseteq \mathbb{R}^{n} \times \mathbb{R}^{m}$ to $\mathbb{R}^{n}$ is defined as

$$
\operatorname{proj}_{\mathbb{R}^{n}}(P):=\left\{x \in \mathbb{R}^{n}:(x, y) \in P \text { for some } y \in \mathbb{R}^{m}\right\} \text {. }
$$

By standard results from polyhedral theory (cf. [5]), the projection of the polyhedron $\mathscr{P}$, which is the closure of the set of solutions of our system of linear inequalities, to $\mathbb{R}^{n \cdot|\Theta|}$ can be written as

$$
\operatorname{proj}_{\mathbb{R}^{n \cdot \Theta \mid}}(\mathscr{P}):=\left\{x \in \mathbb{R}^{n \cdot|\Theta|}: r_{\lambda}^{T}(b-A x) \geq 0 \text { for all } \lambda \in \Lambda\right\},
$$

where $\left\{r_{\lambda}\right\}_{\lambda \in \Lambda}:=$ extreme. $\operatorname{rays}(Q)$ is the finite set of extreme rays of the polyhedron $Q:=\left\{v \in \mathbb{R}_{+}^{k}\right.$ : $\left.v^{T} B=0\right\}$. In particular, $\operatorname{proj}_{\mathbb{R}^{n \cdot|\Theta|}}(\mathscr{P})$ is a polyhedron.

Lemma 1. Let $P_{1} \subseteq \mathbb{R}^{n}$ and $P_{2} \subseteq \mathbb{R}^{m}$ be polyhedra and $P=P_{1} \times P_{2}$. Then

$$
\text { extreme. } \operatorname{rays}(P)=\text { extreme. } \operatorname{rays}\left(P_{1}\right) \times \text { extreme. } \operatorname{rays}\left(P_{2}\right) \text {. }
$$

Lemma 2. Let $K^{r i}$ denote the relative interior of a convex set $K$. If $P \subseteq \mathbb{R}^{n} \times \mathbb{R}^{m}$ is a polyhedron, then $\operatorname{proj}_{\mathbb{R}^{n}}(P)^{r i}=\operatorname{proj}_{\mathbb{R}^{n}}\left(P^{r i}\right)$. Moreover, if $P=P_{1} \times P_{2}$ for polyhedra $P_{1} \subseteq \mathbb{R}^{n}$ and $P_{2} \subseteq \mathbb{R}^{m}$, then $P^{r i}=P_{1}^{r i} \times P_{2}^{r i}$.

The following proposition is crucial for the proof of Proposition 1 We use $\operatorname{ker}(C)$ to denote the kernel $\{z: C z=0\}$ of a matrix $C$.

Proposition 2. Each entry of an extreme ray $r_{\lambda}$ of the polyhedron $Q$ has encoding length polynomial in the input size of the Strong Implementability Problem. 
Proof. $Q$ can be written as $Q=\left\{v \in \mathbb{R}_{+}^{k}: B^{T} v=0\right\}=\operatorname{ker}\left(B^{T}\right) \cap \mathbb{R}_{+}^{k}$, where the matrix $B^{T}$ has the form

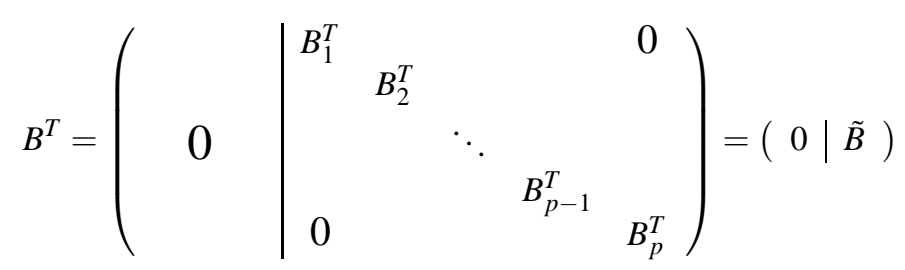

Here, $B_{j} \in \operatorname{Mat}\left(\left(1+\left|\Theta_{i_{\alpha_{j}}}\right|\right) \times\left|\Theta_{-i_{\alpha_{j}}}\right|, \mathbb{Q}\right)$ is the coefficient matrix of the $\left|\Theta_{-i_{\alpha_{j}}}\right|$ variables $\bar{P}_{i_{\alpha_{j}}}^{\alpha_{j}}\left(\theta_{-i_{\alpha_{j}}}\right)$, $\theta_{-i_{\alpha_{j}}} \in \Theta_{-i_{\alpha_{j}}}$, for the bad equilibrium $\alpha_{j}$ in the inequalities (3) and (4) corresponding to $\alpha_{j}$. Hence, we can write $Q$ as

$$
Q=\mathbb{R}_{+}^{q+m \cdot \Sigma_{i=1}^{n}\left|\Theta_{i}\right|^{2}} \times\left(\operatorname{ker}(\tilde{B}) \cap \mathbb{R}_{+}^{p+\sum_{\alpha \text { bad equ. }}\left|\Theta_{i \alpha}\right|}\right)
$$

where $\tilde{B} \in \operatorname{Mat}\left(l \times\left(p+\sum_{\alpha \text { bad equ. }}\left|\Theta_{i_{\alpha}}\right|\right), \mathbb{Q}\right)$ is the submatrix of $B^{T}$ obtained by deleting the $q+m$. $\sum_{i=1}^{n}\left|\Theta_{i}\right|^{2}$ zeros at the beginning of each line as shown in (5). Hence, by Lemma11 every extreme ray $r_{\lambda}$ of $Q$ is of the form $r_{\lambda}=\left(e_{i}, r\right)$, where $e_{i}$ is a unit vector in $\mathbb{R}^{q+m \cdot \sum_{i=1}^{n}\left|\Theta_{i}\right|^{2}}$ (which are the extreme rays of $\left.\mathbb{R}_{+}^{q+m \cdot \sum_{i=1}^{n}\left|\Theta_{i}\right|^{2}}\right)$ and $r$ is an extreme ray of $\operatorname{ker}(\tilde{B}) \cap \mathbb{R}_{+}^{p+\sum_{\alpha \text { bad equ. }}\left|\Theta_{i \alpha}\right|}$.

To obtain a description of the extreme rays of $\operatorname{ker}(\tilde{B}) \cap \mathbb{R}_{+}^{p+\sum_{\alpha \text { bad equ. }}\left|\Theta_{i \alpha}\right|}$, note that $\tilde{B}$ is the direct sum of the matrices $B_{1}^{T}, \ldots, B_{p}^{T}$, so we have

$$
\operatorname{ker}(\tilde{\boldsymbol{B}}) \cap \mathbb{R}_{+}^{p+\sum_{\alpha \text { bad equ. }}\left|\Theta_{i \alpha}\right|}=\bigoplus_{j=1}^{p}\left(\operatorname{ker}\left(B_{j}^{T}\right) \cap \mathbb{R}_{+}^{1+\left|\Theta_{i \alpha_{j}}\right|}\right) .
$$

Thus, again by Lemma 11, every extreme ray $r_{\lambda}$ of $Q$ is of the form $r_{\lambda}=\left(e_{i}, r_{1}, \ldots, r_{p}\right)$, where $e_{i}$ is a unit vector in $\mathbb{R}^{q+m \cdot \sum_{i=1}^{n}\left|\Theta_{i}\right|^{2}}$ and $r_{j} \in \mathbb{R}^{1+\left|\Theta_{i \alpha_{j}}\right|}$ is an extreme ray of $Q_{j}:=\operatorname{ker}\left(B_{j}^{T}\right) \cap \mathbb{R}_{+}^{1+\left|\Theta_{i \alpha_{j}}\right|}$ for every $j=1, \ldots, p$. Hence, it just remains to show that, for every $j=1, \ldots, p$, each entry of an extreme ray $r_{j}$ of $Q_{j}$ has polynomial encoding length. This follows by writing $Q_{j}=\left\{v \in \mathbb{R}_{+}^{1+\left|\Theta_{i \alpha_{j}}\right|}: B_{j}^{T} v=0\right\}$ and the fact that the encoding length of $B_{j}$ is polynomial for every $j$.

Proposition 3. The facet complexity of proj $\mathbb{R}_{\mathbb{R}^{n \cdot \Theta \mid}}(\mathscr{P})$ is polynomial in the encoding length of the input of Strong Implementability.

Proof. As already stated, we have

$$
\begin{aligned}
\operatorname{proj}_{\mathbb{R}^{n \cdot \Theta \mid}}(\mathscr{P}) & =\left\{x \in \mathbb{R}^{n \cdot|\Theta|}: r_{\lambda}^{T}(b-A x) \geq 0 \text { for all } \lambda \in \Lambda\right\} \\
& =\left\{x \in \mathbb{R}^{n \cdot|\Theta|}:\left(r_{\lambda}^{T} A\right) x \leq r_{\lambda}^{T} b \text { for all } \lambda \in \Lambda\right\}
\end{aligned}
$$

where extreme. rays $(Q)=\left\{r_{\lambda}\right\}_{\lambda \in \Lambda}$ is the finite set of extreme rays of $Q$. Hence, the claim follows if we show that each inequality $\left(r_{\lambda}^{T} A\right) x \leq r_{\lambda}^{T} b$ is of polynomial encoding length. To this end, consider the inequality $\left(r_{\lambda}^{T} A\right) x \leq r_{\lambda}^{T} b$ for a fixed $\lambda \in \Lambda$. By Proposition 2, each entry of the extreme ray $r_{\lambda}$ has polynomial encoding length. Since each entry of $b$ has polynomial encoding length as well, the value $r_{\lambda}^{T} b \in \mathbb{Q}$ is a sum of exponentially many values of polynomial encoding length, which is again of polynomial encoding length. Similarly, since each entry of the matrix $A$ has polynomial encoding length, each entry of the vector $r_{\lambda}^{T} A$ has polynomial encoding length, which implies that the whole vector $r_{\lambda}^{T} A$ is of polynomial encoding length since the vector is of polynomial size. Thus, the encoding length of $\left(r_{\lambda}^{T} A\right) x \leq r_{\lambda}^{T} b$ is polynomial. 
Proof of Proposition $\square$ Let eq $(\mathscr{P})$ (the equality set of $\mathscr{P}$ ) denote the set of indices of inequalities in $A x+B y \leq b$ that are satisfied with equality for all points in $\mathscr{P}$. It is enough to prove the existence of a point $(\bar{x}, \bar{y})$ in $\mathscr{P}^{\text {ri }}$ such that each component of $(\bar{x}, \bar{y})$ has polynomial encoding length: Such a point $(\bar{x}, \bar{y})$ has to satisfy all inequalities with indices not in eq $(\mathscr{P})$ with strict inequality. Moreover, since the original system (where the inequalities (1) and (3) are strict) has a solution, we know that all indices of the inequalities (1) and (3) are not in eq $(\mathscr{P})$. Hence, $(\bar{x}, \bar{y})$ satisfies all the inequalities (1) and (3) with strict inequality, i.e., it is a solution of the original system.

By Proposition $3, \operatorname{proj}_{\mathbb{R}^{n \cdot \Theta \mid}}(\mathscr{P})$ is a nonempty polyhedron of polynomial facet complexity. Using standard results from polyhedral theory (cf. [1, Thm. 6.5.5]), this implies the existence of a point $\bar{x}$ of polynomial encoding length in $\operatorname{proj}_{\mathbb{R}^{n \cdot \Theta \mid}}(\mathscr{P})^{\mathrm{ri}}$. In particular, each component of $\bar{x}$ has polynomial encoding length. Moreover, by Lemma 2, we have $\bar{x} \in \operatorname{proj}_{\mathbb{R}^{n \cdot|\Theta|}}\left(\mathscr{P}^{\mathrm{ri}}\right)$, so we can choose $y_{0} \in \mathbb{R}^{l}$ such that $\left(\bar{x}, y_{0}\right) \in \mathscr{P}$ ri i.e., $y_{0}$ is a solution of the system $A \bar{x}+B y \leq b \Leftrightarrow B y \leq b-A \bar{x}$ and all inequalities with indices not in eq $(\mathscr{P})$ are satisfied strictly for $y=y_{0}$. Writing $\tilde{\mathscr{P}}:=\left\{y \in \mathbb{R}^{l}: B y \leq b-A \bar{x}\right\}$, we have eq $(\tilde{\mathscr{P}})=\mathrm{eq}(\mathscr{P})$ : The inclusion eq $(\tilde{\mathscr{P}}) \supseteq \mathrm{eq}(\mathscr{P})$ follows from the definition of $\tilde{\mathscr{P}}$ and the other inclusion follows since $y_{0} \in \tilde{\mathscr{P}}$ satisfies all inequalities with indices not in eq $(\mathscr{P})$ with strict inequality. The matrix $B$ has the form

$$
B=\left(\begin{array}{cccc}
0 & 0 & 0 & 0 \\
B_{1} & & & 0 \\
& B_{2} & & \\
& & \ddots & \\
0 & & & B_{p}
\end{array}\right) .
$$

Hence, the system $B y \leq b-A \bar{x}$ decomposes into $p$ smaller systems $B_{j} y^{j} \leq b^{j}-A^{j} \bar{x}^{j}, j=1, \ldots, p$, where $b^{j}, A^{j}, \bar{x}^{j}$ denote the parts of $b, A$ and $\bar{x}$, respectively, corresponding to the lines of the system containing the submatrix $B_{j}$. Writing

$$
\tilde{\mathscr{P}}_{j}:=\left\{y^{j} \in \mathbb{R}^{\left|\Theta_{-i \alpha_{j}}\right|}: B_{j} y^{j} \leq b^{j}-A^{j} \bar{x}^{j}\right\}
$$

we have $\tilde{\mathscr{P}}=\tilde{\mathscr{P}}_{1} \times \cdots \times \tilde{\mathscr{P}}_{p}$, and each $\tilde{\mathscr{P}}_{j}$ is nonempty because $\tilde{\mathscr{P}}$ is nonempty.

Since $B_{j} \in \operatorname{Mat}\left(\left(1+\left|\Theta_{i_{\alpha_{j}}}\right|\right) \times\left|\Theta_{-i_{\alpha_{j}}}\right|\right)$ is of polynomial size for every $j$, and each entry of $A, B, b$, and $\bar{x}$ has polynomial encoding length, the facet complexity of each polyhedron $\tilde{\mathscr{P}}_{j}$ is polynomial. Thus, again by standard results from polyhedral theory, the relative interior of each $\tilde{\mathscr{P}}_{j}$ contains a point $\tilde{y}^{j}$ of polynomial encoding length.

If we now define $\bar{y}:=\left(\tilde{y}^{1}, \ldots, \tilde{y}^{p}\right)$, all components of the vector $(\bar{x}, \bar{y})$ have polynomial encoding length and we have $\bar{y} \in \tilde{\mathscr{P}}_{1}^{\text {ri }} \times \cdots \times \tilde{\mathscr{P}}_{p}^{\mathrm{ri}}=\tilde{\mathscr{P}}^{\mathrm{ri}}$, where the equality follows by Lemma 2 Hence, $(\bar{x}, \bar{y})$ satisfies $A \bar{x}+B \bar{y} \leq b$ and all inequalities with indices not in $\operatorname{eq}(\tilde{\mathscr{P}})=\mathrm{eq}(\mathscr{P})$ are satisfied with strict inequality, i.e., $(\bar{x}, \bar{y}) \in \mathscr{P}^{\text {ri }}$.

\section{Theorem 5. Strong Implementability $\in$ PSPACE.}

Proof. Assume that the given social choice function $f$ is strongly implementable. Then, by Theorem 4 , there exists an incentive compatible direct revelation mechanism $\Gamma_{(f, P)}$ of polynomial encoding length that satisfies the selective elimination condition. Moreover, for every bad equilibrium $\alpha$ of $\Gamma_{(f, P)}$, the data $\left(i_{\alpha}, h^{\alpha}, \bar{P}_{i_{\alpha}}^{\alpha}, \bar{\theta}_{i_{\alpha}}^{\alpha}\right)$ needed to selectively eliminate $\alpha$ can also be chosen to have polynomial encoding length. Now consider Algorithm 1 on the following page for verifying that $f$ is strongly implementable.

Since all the values $P_{i}(\theta)$ and the data $\left(i_{\alpha}, h^{\alpha}, \bar{P}_{i_{\alpha}}^{\alpha}, \bar{\theta}_{i_{\alpha}}^{\alpha}\right)$ for every bad equilibrium $\alpha$ have polynomial encoding length and every inequality in the system is of polynomial encoding length, Algorithm 11 uses only polynomial space, which proves the claim. 


\begin{abstract}
Algorithm 1.
1. Guess the (polynomially many) values $P_{i}(\theta)$.

2. For every strategy profile $\alpha=\left(\alpha_{1}, \ldots \alpha_{n}\right)$ in the mechanism $\Gamma_{(f, P)}$ do:

- Check whether $\alpha$ is an equilibrium by going through the inequalities (2) corresponding to $\alpha$ one by one.

- If $\alpha$ is not an equilibrium, we have already found $\left(i_{\alpha}, \theta_{i_{\alpha}}^{\alpha}, \bar{\theta}_{i_{\alpha}}^{\alpha}\right)$ such that the inequality (1) corresponding to $\alpha$ is satisfied in the previous step.

- If $\alpha$ is an equilibrium, check whether $f \circ \alpha=f$ by going through all possible bid vectors $\theta \in \Theta$. If $f \circ \alpha \neq f$, guess the data $\left(i_{\alpha}, h^{\alpha}, \bar{P}_{i_{\alpha}}^{\alpha}, \bar{\theta}_{i_{\alpha}}^{\alpha}\right)$ of polynomial encoding length needed to selectively eliminate $\alpha$ and check the inequalities (3) and (4) corresponding to $\alpha$ one by one.
\end{abstract}

As a byproduct of Theorem 4, we obtain the following result on the size of the payments needed to strongly implement a social choice function:

Corollary 1. If the social choice function $f: \Theta \rightarrow X$ is strongly implementable, it can be strongly implemented by an augmented revelation mechanism $\Gamma=\left(S_{1}, \ldots, S_{n}, g, P\right)$ in which each payment $P_{i}(s), s \in S$, $i \in N$, has polynomial encoding length.

Proof. If $f$ is strongly implementable, Theorem 4 implies the existence of an incentive compatible direct revelation mechanism $\Gamma_{\left(f, P^{\prime}\right)}^{\prime}$ of polynomial encoding length that satisfies the selective elimination condition and, for every bad equilibrium $\alpha$ of $\Gamma_{\left(f, P^{\prime}\right)}^{\prime}$, the data $\left(i_{\alpha}, h^{\alpha}, \bar{P}_{i_{\alpha}}^{\alpha}, \bar{\theta}_{i_{\alpha}}^{\alpha}\right)$ needed to selectively eliminate $\alpha$ can also be chosen to have polynomial encoding length. In particular, all payments and "elimination payments" $\bar{P}_{i_{\alpha}}^{\alpha}$ are of polynomial encoding length. As shown in the proof of the sufficiency part of Theorem 3 given in [3], we can then obtain an augmented revelation mechanism as in the claim from $\Gamma_{\left(f, P^{\prime}\right)}^{\prime}$ by a sequence of at most $|\Theta|^{|\Theta|} \leq 2^{|\Theta|^{2}}$ augmentations. In each step, one of the at most $|\Theta|^{|\Theta|}$ bad equilibria is eliminated without introducing any new equilibria. The payments after each augmentation are given by the $\bar{P}_{i_{\alpha}}^{\alpha}$ and the payments from the previous step, which are only changed by additive terms of polynomial encoding length. Since there are at most $|\Theta|^{|\Theta|} \leq 2^{|\Theta|^{2}}$ augmentation steps and each of the initial payments in $\Gamma_{\left(f, P^{\prime}\right)}^{\prime}$ is also of polynomial encoding length, this implies that each payment $P_{i}(s)$ in the final augmented revelation mechanism that strongly implements $f$ is of polynomial encoding length.

\title{
3 The Single-Agent Scenario
}

In this section, we show that, in the case of a single agent, Strong Implementability can be decided in polynomial time via linear programming.

Definition 9. Given a single-agent mechanism $\Gamma=(S, g, P)$ and a type $\theta \in \Theta$ of the agent, the utility from a bid $s \in S$ for the agent is defined as $U^{\Gamma}(s \mid \theta):=V(g(s), \theta)+P(s)$. A strategy $\alpha: \Theta \rightarrow S$ of the agent in the mechanism $\Gamma$ is an equilibrium strategy (or simply an equilibrium) if $\alpha(\theta)$ maximizes the utility of the agent for every $\theta \in \Theta$, i.e., if $U^{\Gamma}(\alpha(\theta) \mid \theta) \geq U^{\Gamma}(\bar{s} \mid \theta)$ for all $\theta \in \Theta, \bar{s} \in S$.

Note that this definition is just the special case of Definition 2 where there is only a single agent. Incentive compatibility of a direct revelation mechanism and strong implementation of a social choice function are defined as before. The Strong Implementability Problem for a single agent (Single-Agent Strong Implementability) is simply the special case of the Strong Implementability Problem with the number $n$ of agents equal to one. Note that the probability distribution $p$ is not needed for a single agent. 
As Single-Agent Strong Implementability is a special case of Strong Implementability, the characterization from Theorem 3 holds in the single-agent scenario as well. However, the definition of selective elimination implies that, with just one agent, selective elimination of a bad equilibrium is never possible. Consequently, a single-agent direct revelation mechanism $\Gamma_{(f, P)}$ satisfies the selective elimination condition if and only if it has no bad equilibria at all and we obtain the following result:

Theorem 6. A social choice function $f: \Theta \rightarrow X$ in the single-agent scenario is strongly implementable if and only if there exists an incentive compatible direct revelation mechanism $\Gamma_{(f, P)}$ without bad equilibria.

We will now show how we can use Theorem 6 to decide Single-Agent Strong Implementability in polynomial time. In the case of a single agent, a bad equilibrium in a direct revelation mechanism is simply a strategy $\alpha: \Theta \rightarrow \Theta$ of the agent such that $\alpha(\theta)$ maximizes the utility of the agent for every $\theta \in \Theta$ and such that $f(\theta) \neq f(\alpha(\theta))$ for at least one $\theta \in \Theta$. Hence, an incentive compatible direct revelation mechanism $\Gamma_{(f, P)}$ has no bad equilibrium if and only if we have

$$
V\left(f\left(\theta^{\prime}\right), \theta\right)+P\left(\theta^{\prime}\right)<V(f(\theta), \theta)+P(\theta)
$$

for all $\theta, \theta^{\prime} \in \Theta$ with $f(\theta) \neq f\left(\theta^{\prime}\right)$. This implies that the possible payment schemes $P$ of an incentive compatible direct revelation mechanism $\Gamma_{(f, P)}$ satisfying the selective elimination condition correspond to the solutions of the following system in the variables $P(\theta)$ for $\theta \in \Theta$ :

$$
\begin{aligned}
V\left(f\left(\theta^{\prime}\right), \theta\right)+P\left(\theta^{\prime}\right) & <V(f(\theta), \theta)+P(\theta) \quad \forall \theta, \theta^{\prime} \in \Theta \text { with } f(\theta) \neq f\left(\theta^{\prime}\right) \\
V(f(\bar{\theta}), \theta)+P(\bar{\theta}) & \leq V(f(\theta), \theta)+P(\theta) \quad \forall \theta, \bar{\theta} \in \Theta
\end{aligned}
$$

Here, the inequalities (7) encode incentive compatibility of the mechanism and, as discussed above, the strict inequalities (6) encode that the mechanism has no bad equilibrium. The polyhedron $\mathscr{P}$ defined by this system when all strict inequalities are replaced by non-strict inequalities has polynomial facet complexity, since the system only contains polynomially many variables (and inequalities) and coefficients of polynomial encoding length. Thus, we can check in polynomial time whether the polyhedron contains a relative interior point corresponding to a solution of the original system with strict inequalities in (6). Hence, we obtain the following result:

Theorem 7. Single-Agent Strong Implementability $\in P$.

In fact, the above system shows that Single-Agent Strong Implementability can be decided via linear programming.

\section{References}

[1] M. Grötschel, L. Lovász \& A. Schrijver (1988): Geometric Algorithms and Combinatorial Optimization. Springer.

[2] A. Mas-Colell, M. D. Whinston \& J. R. Green (1995): Microeconomic Theory. Oxford University Press.

[3] D. Mookherjee \& S. Reichelstein (1990): Implementation Via Augmented Revelation Mechanisms. Review of Economic Studies 57(3), pp. 453-475.

[4] R. Müller, A. Perea \& S. Wolf (2007): Weak Monotonicity and Bayes-Nash Incentive Compatibility. Games and Economic Behavior 61, pp. 344-358.

[5] G. L. Nemhauser \& L. A. Wolsey (1988): Integer and Combinatorial Optimization. Wiley-Interscience, New York.

[6] N. Nisan \& A. Ronen (2001): Algorithmic Mechanism Design. Games and Economic Behavior 35, pp. 166196.

[7] M. J. Osborne \& A. Rubinstein (1994): A Course in Game Theory. MIT Press. 